\title{
An Investigation into Failure of Starter Solenoid Spring
}

\author{
Rajeshkumar Ramasamy*, Neelakandan V, Ganesan T and Praveen CR \\ Comstar Automotive Technologies, Keelakaranai Village, Malrosapuram Post, Maraimalai Nagar, Tamil Nadu 603204, India
}

\begin{abstract}
The Plunger return spring of Starter Solenoid is prematurely failed during service. The said failure is presumed to be associated with the combined effects of wear, cold drawn micro crack and tool mark formed during the service. In-order to understand the cause of the failure, the present paper aims to investigate the spring failure at $203 \mathrm{k}$ cycle, by Chemical analysis, Scanning Electron Microscope and Vickers hardness assessment. The test reports from the above said assessments disclose surface irregularities and does not show any noticeable change in chemical composition and hardness. The studies reveal that, the wear scratches are formed due to non-uniform contact of plunger components, which cause the surface crack in the internal dia of the spring. Further, Oxide inclusions act as crack initiation sites, where Crack initiation and propagation route are clearly observed in the Scanning Electron Microscope images. This paper, further discusses the methods to improve the spring life.
\end{abstract}

Keywords: Solenoid spring; Hardness; Microstructure; Oxides inclusion; Crack initiation

\section{Introduction}

The plunger of the solenoid is connected concentrically to a spring, which is compressed when coil near the plunger is electrically connected. The reverse action, disconnects the plunger electrically. The spring is designed in such a way, to withstand the compression and tension created during the lifecycle, to ensure the completion of $300 \mathrm{~K}$ cycles. The schematic representation of plunger return spring in solenoid and its configuration is shown in Figures 1 and 2.

The recurrent loading of the spring, results in fatigue. Predominantly, the surface roughness and inclusions are the stress raisers of the spring. In-order to minimize the effects of stress raisers, factors such as lack of microstructure, manufacturing quality and material property must be negligible [1].

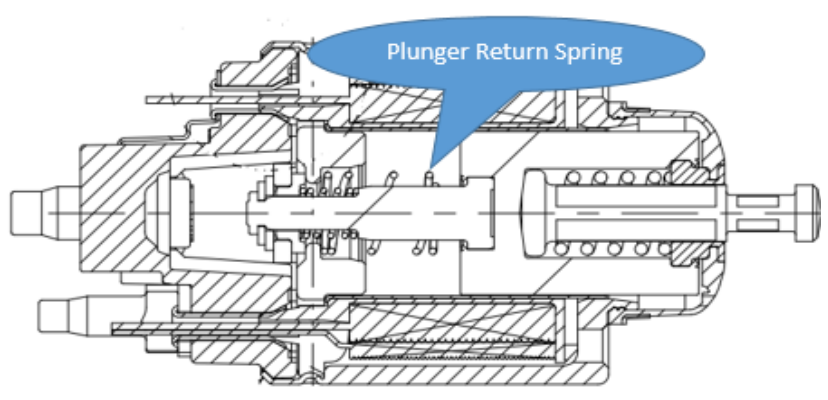

Figure 1: Assembled view of plunger return spring.

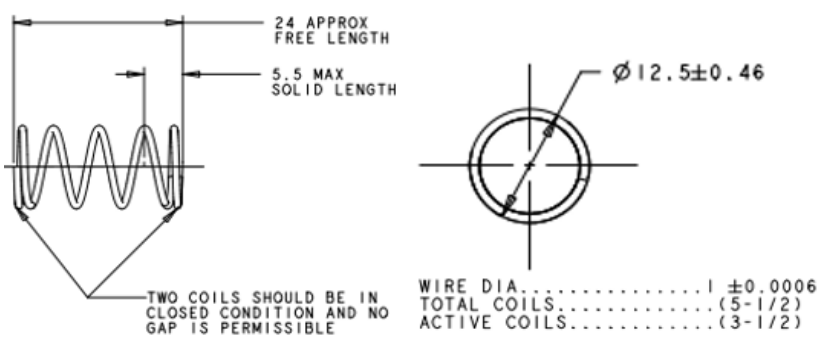

Figure 2: Configuration of the spring.
During service, the inner surface of any active coil may experience the maximum stress, which leads to imperfections. This imperfection may turn into fatigue crack initiation if the surface morphology is irregular [2].

\section{Experimental Work}

The spring is manufactured from the material called steel wire Music spring ASTM A228. The property of the material is to withstand the temperature ranging from $-150^{\circ} \mathrm{C}$ to $200^{\circ} \mathrm{C}$. The plunger spring used in solenoid and its failure configuration is shown in Figures $3 \mathrm{a}$ and $3 \mathrm{~b}$. In order to prevent the material from oxidation and corrosion during elevated (high) temperature, it is subjected to Zinc electroplating. For visual examination the sample is cleaned with acetone, which is done prior to metallographic inspection. Then, the fractured surface of the spring is analyzed using Scanning Electron Microscope. The samples are prepared from the fractured spring for microstructural analysis, which are further polished and etched with $2 \%$ nital reagent $(2 \mathrm{ml}$

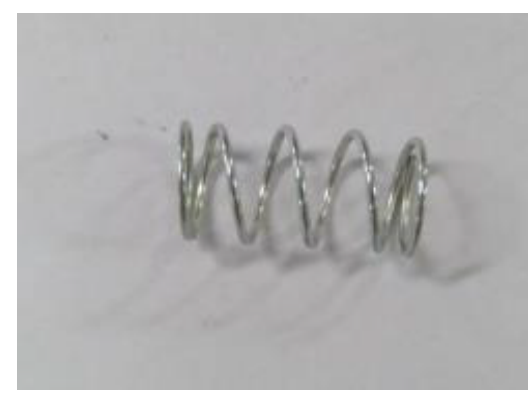

Figure 3a: Actual Spring

*Corresponding author: Rajeshkumar Ramasamy, Comstar Automotive Technologies, Keelakaranai Village, Malrosapuram Post, Maraimalai Nagar, Tamil Nadu 603204, India, Tel: 044-3747 3700; E-mail: rrajesh@comstarauto.com

Received August 20, 2018; Accepted October 15, 2018; Published October 25 2018

Citation: Ramasamy R, Neelakandan V, Ganesan T, Praveen CR (2018) An Investigation into Failure of Starter Solenoid Spring. J Material Sci Eng 7: 491. doi: 10.4172/2169-0022.1000491

Copyright: (c) 2018 Ramasamy R, et al. This is an open-access article distributed under the terms of the Creative Commons Attribution License, which permits unrestricted use, distribution, and reproduction in any medium, provided the original author and source are credited. 
$\mathrm{HNO}_{3}$ in $98 \mathrm{ml}$ ethyl alcohol). The Vickers micro hardness assessment is carried out to find the variation in the sample before and after the service. Lastly, the Optical Spectrometer test is done to evaluate the element concentration on samples before and after the service.

\section{Results and Discussion}

\section{Visual observation}

The Fracture edge and crack initiation location is shown in Figure 4a. From the Figure $4 \mathrm{~b}$, it is evident that, the samples possess visible tool mark and deep scar, which also shows the crack initiation point. The fracture edge is extended at an angle of $45^{\circ}$ on one side of the wire axis and the other side extending half way through the wire thickness following the tool mark. The crack grows as spiral around the axis surface, which is typically known as torsional fatigue as shown in Figure 4c [3-5].

The fatigue origin is identified at the inner surface of the active coil, which is occurred due to contact and friction of inner surface of the spring with plunger. The material of the plunger and spring is designed to withstand the contact wear to improve the life. But, the irregular flattened surface of the spring and non-uniform contact of plunger and spring, creates stress singularity and high friction at particular location [5]. The fatigue crack originated at rubbing surface of two mating parts, where the wear scar and high stress are experienced.

\section{Chemical analysis}

The chemical analysis of the sample before and after the service is completed and stated in Table 1. The samples for the test are polished and flat. The test equipment generates electrical energy in the form of spark between sample and electrode, where the extracted emission spectrum from the targeted elements illustrates the chemical composition. It is clearly noted that, there is no significant change in elemental fraction and the material is in suitable condition for service.

\section{Hardness assessment}

The Table 2 represents the Core Vickers hardness of the samples after service. The hardness of fractured samples are equivalent with Music wire ASTM A228 spring criterion [4].

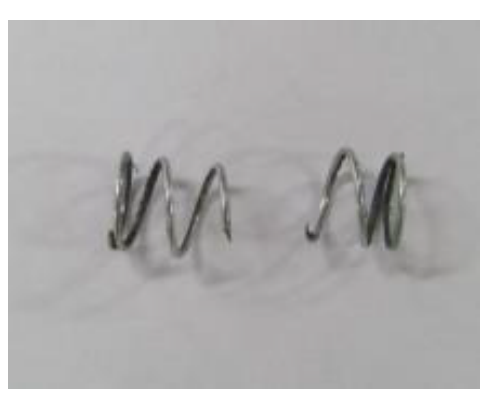

Figure 3b: Failure spring

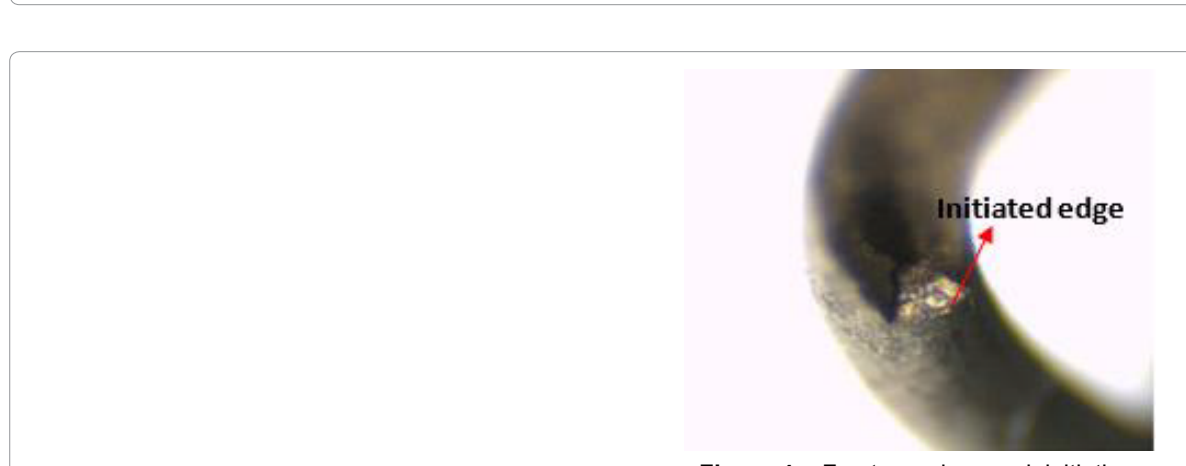

Figure 4a: Fracture edge crack initiation.

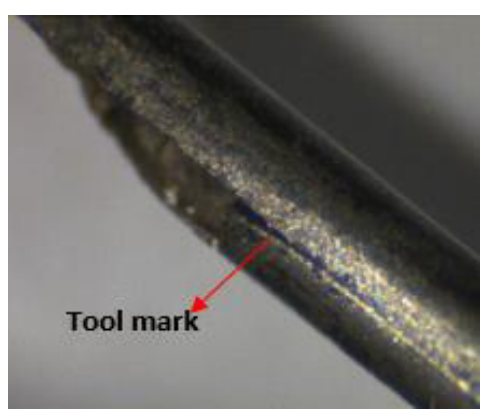

Figure 4b: Tool mark and crack initiation. 


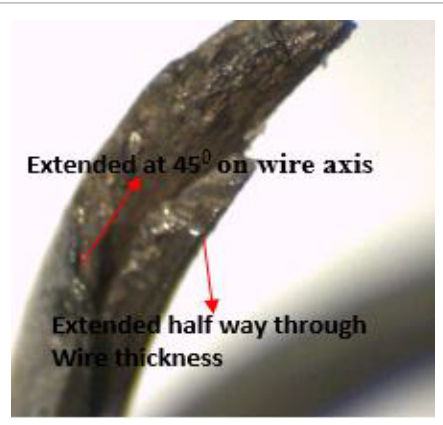

Figure 4c: Crack propagation.

\begin{tabular}{|l|c|c|c|c|c|}
\hline Elements In Wt.\% & C & Mn & Si & Fe & S \\
\hline Composition of ASTM A228 Music Wire & $0.7-1.0$ & $0.2-0.6$ & $0.1-0.3$ & Max0.025 & Max 0.030 \\
\hline Observed In Testing & 0.74 & 0.35 & 0.19 & 0.02 & 0.02 \\
\hline
\end{tabular}

Table 1: Chemical Composition of failed part.

\section{Failed Component}

Vickers Harness Number VHN -542, 569, 550 at core

Table 2: Hardness Measurement.

\section{SEM observation}

Wear Scar and Micro crack are observed at inner surface of the active coil of the spring as in Figure 5a, the wear scar and the crack propagation path act as stress raiser resulting in catastrophic failure. It is confirmed that, the spring and plunger had non-uniform contact and experienced vigorous deformation. The crack is continued to propagate to the core in the direction of inner coil dia towards midway of the wire center as shown in Figure $5 \mathrm{~b}$ and $5 \mathrm{c}$. The crack might have elevated to the core instead of the surface because of some oxide inclusion at path of propagation. It is exemplified by the deep pull out of surface Figure $5 \mathrm{c}$. From the core, the crack propagates in both longitudinal and radial direction as shown in Figure 5d.

It is observed that, all region of fracture have cleavage patterns, which confirms that, the material failed in intergranular brittle manner as in Figure 5e and 5f. In case, if it would have been due to ductile failure, the samples must have shown ductile dimple pattern, instead of cleavage pattern [6]. The crack initiated deforms the surface of protective layer, which led the elements to oxidize and experience corrosion. Corrosion pit is observed at crack tip as shown in Figure 5c, which is the reason for crack elevation towards core.

\section{Recommendations}

The failure investigation of spring for solenoid plunger return is carried out through Chemical analysis, Microstructure Examination and Hardness assessment. Based on the test results the following conclusions are derived,

1. Fatigue failure occurred in the spring is by the combined effects of wear, tool mark and corrosion pits associated with stress singularities at the contact surface of inner coil surface and plunger.

2. Once the crack initiated, it is propagated to core with direction of $45^{\circ}$ to the tensile axis of the wire.

3. The crack is propagated by the force of maximum compressive stress.
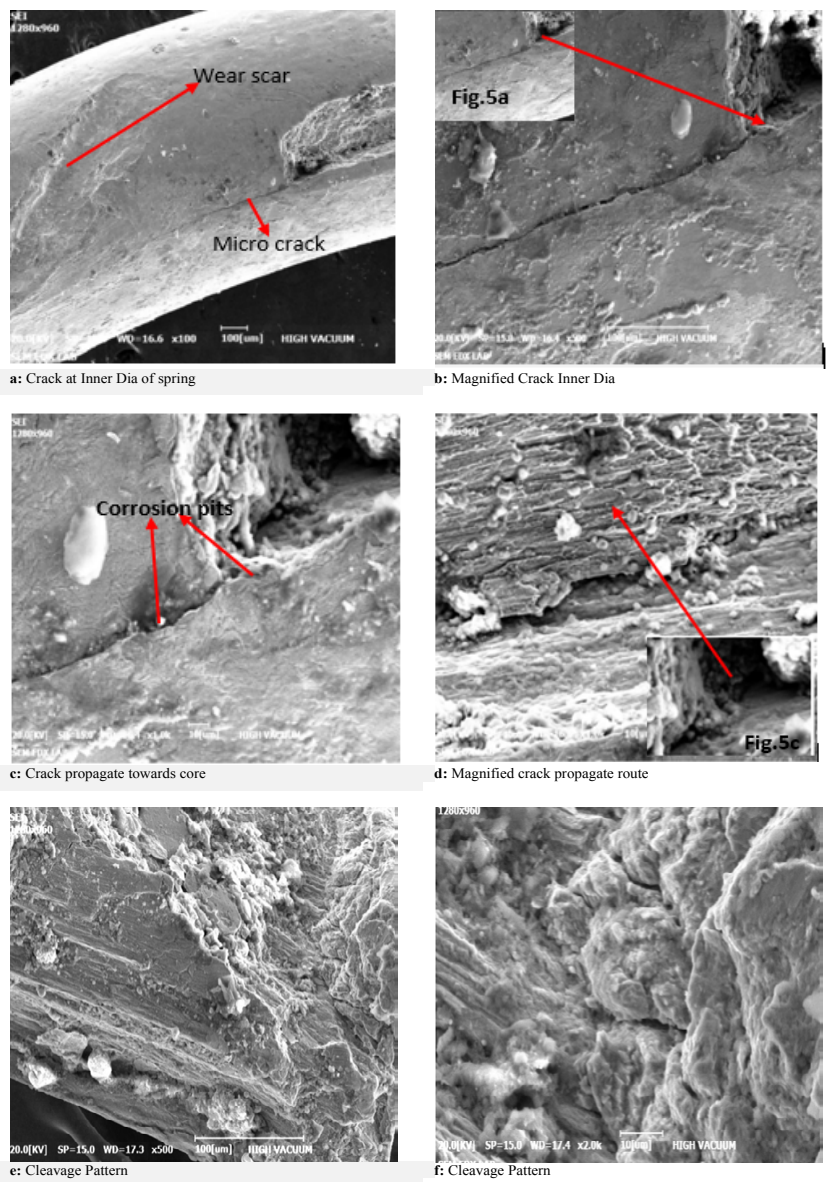

Figure 5: SEM images of crack initiation and propagation.

4. It is strongly recommended to have uniform contact between spring and plunger in order to avoid stress singularities and frictional wear.

5. The materials should be treated for secondary process to improve the wear resistance and to reduce the surface integrity.

6. The flattened level in closed end of the spring and flattened 
Citation: Ramasamy R, Neelakandan V, Ganesan T, Praveen CR (2018) An Investigation into Failure of Starter Solenoid Spring. J Material Sci Eng 7: 491. doi: 10.4172/2169-0022.1000491

Page 4 of 4

surface required for spring base, plays an important role in stress singularities and frictional wear.

\section{References}

1. Vukelic G, Brcic M (2016) Failure analysis of a motor vehicle coil spring Procedia Structural Integrity 2: 2944-2950.

2. Wahl AM (1984) Mechanical springs. McGraw-Hill Book Company.

3. Zhu $Y$, Wang $Y$, Huang $Y(2014)$ Failure analysis of a helical compression spring for a heavy vehicle's suspension system. Case Studies in Engineering Failure Analysis 2: 169-173.

4. Miller KJ (1970) Cyclic behavior of materials. Journal of Strain Analysis 5: 133136.

5. Esklul K (1994) Handbook of case histories in failure analysis. ASM International.

6. ASM Handbook Fractography (1987) ASM International (9thedn). 